\title{
Estrongiloidosis como causa de hemorragia digestiva baja
}

\author{
Patricia Paredes, Janeth Atiaja y Santiago Lanas
}

\section{Strongyloidiasis as cause of lower gastrointestinal bleeding}

Intestinal parasites are a common pathology in the pediatric practice, constituting a major public health problem. Currently their epidemiology, especially in countries like Ecuador where there are populations and communities where health services are scarce or difficult to access. We report the case of a child with chronic grade 3 malnutrition, from Sarayacu province of Pastaza, Ecuador an endemic area in several parasitosis. The clinical features were lower gastrointestinal bleeding added to chronic anemia and eosinophilia. The patient underwent surgery, colectomy right after finding at colonoscopy mass at the small intestine and colon, which ends with the symptoms. We get to the subsequent diagnosis histopathological analysis in which found peritoneal invasion of Strongyloides stercoralis larvae filariform. This article shows a general perspective on the gastrointestinal bleeding in pediatrics and relevant data about the strongyloidiasis.

Key words: Strongyloides, gastrointestinal bleeding, anemia.

Palabras clave: Strongyloides, hemorragia digestiva, anemia.
Hospital General del Seguro Social-Ambato, Ecuador. Servicio de Pediatría (PP) Servicio de Gastroenterología (JA). Universidad Técnica de Ambato (SL).

Los autores declaran no tener conflicto de interés. Sin financiamiento.

Recibido: 3 de agosto de 2015 Aceptado: 13 de septiembre de 2016

Correspondencia a: Patricia Paredes L. patypediatra68@gmail.com

\section{Introducción}

\section{$\mathrm{L}$}

a hemorragia digestiva es una patología grave en niños ya que la pérdida de sangre desde el tracto gastrointestinal, aunque sea leve, puede deteriorar rápidamente al paciente ${ }^{1}$. Puede resultar difícil establecer la causa de la hemorragia, es por ello, que el diagnóstico diferencial debe enfocarse según la presentación clínica y edad del paciente ${ }^{2}$.

La hemorragia digestiva baja constituye aproximadamente $0,3 \%$ de las visitas a las unidades de emergencias pediátricas. De éstas, sólo 4\% implican condiciones que comprometen la vida del paciente. La etiología varía según la edad, sin embargo, las causas infecciosas (ya sea bacterianas, virales o parasitarias), pueden estar involucradas dentro de cualquier grupo etario ${ }^{1}$.

Considerando que aproximadamente $25 \%$ de la población mundial está infectada por helmintos intestinales ${ }^{3}$, ${ }^{1}$ as causas infecciosas deben ser investigadas dentro del estudio del paciente con hemorragia digestiva. A pesar que la etiología parasitaria es infrecuente, el diagnóstico diferencial debe incluir anquilostomiasis, ascariasis, infección amebiana, infecciones por Strongyloides, especialmente en nuestro medio ${ }^{2}$.

Strongyloides stercoralis es un geohelminto que está considerado por la OMS como causa de las enfermedades menospreciadas (NTDs), específicamente dentro de las "soil- transmitted helmintiasis"11. La estrongiloidosis es la infección producida por el nemátodo del género Strongyloides. La infección grave resulta del aumento de la generación de larvas filariformes, lo que ocurre en situaciones de inmunocompromiso como la terapia con corticoesteroides, $\mathrm{u}$ otros inmunosupresores, neoplasias hematológicas o desnutrición ${ }^{3,4}$.

\section{Caso clínico}

Preescolar de 3 años 10 meses procedente y residente de Sarayacu, provincia de Pastaza, Ecuador. Ubicada a orillas del río Bobonaza, los habitantes tienen el hábito cultural de caminar descalzos. El niño nació en su domicilio y el parto fue atendido por su abuela. Como antecedente destaca que el paciente dos años atrás había presentado una enfermedad diarreica que requirió internación y una anemia crónica. Ingresó a nuestro Servicio de Pediatría por un cuadro de tres días de evolución caracterizado por dolor abdominal difuso, tipo cólico, de moderada intensidad. Concomitantemente presentó varios episodios de deposiciones semilíquidas con sangre fresca en escasa cantidad. El cuadro clínico se acompañó de una marcada baja de peso en el último mes y evidente compromiso del estado general.

El examen físico evidenció un niño con signos de desnutrición grave, antropometría (peso y talla Z -2), mal estado general, T $37{ }^{\circ} \mathrm{C}, \mathrm{FC} 110$ por min, FR 23 por min, y oximetría de pulso $93 \%$. La piel presentaba una turgencia disminuida, mucosas orales secas, palidez generalizada y tejido celular subcutáneo disminuido. El examen cardiopulmonar era normal. Al examen abdominal destacaba dolor a la palpación difusa de predominio en mesogastrio, ruidos hidroaéreos aumentados, sin visceromegalia. Extremidades normales, sin edema. En la exploración anal se evidenció una hemorragia activa con sangre roja rutilante. 
Figura 1. En todo el trayecto del colon se observa abundante cantidad de líquido hemático de color café obscuro en la luz y adherido a la mucosa. Servicio de Gastroenterología H. IESS Ambato.

Figura 2. Múltiples pólipos sésiles de 2-4 mm de superficie lisa a $30 \mathrm{~cm}$ del margen anal. Servicio de Gastroenterología H. IESS Ambato.

Figura 3. Masa en intestino delgado. Cortesía Dr. Fausto Rodas. Servicio de Cirugía H. IESS Ambato.

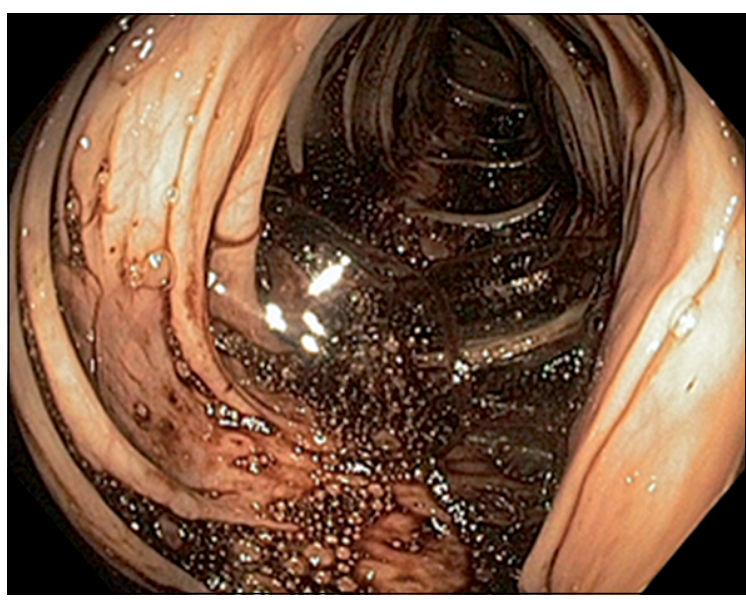

y una observación microscópica directa de deposiciones con ausencia de parásitos y polimorfonucleares, y con abundancia de hematíes (50-60 por campo). La serología para VIH fue negativa.

Con el diagnóstico de una hemorragia digestiva baja, el paciente fue sometido a una laparotomía exploradora encontrándose múltiples masas sugerentes de pólipos. El paciente evolucionó con varios episodios de hematoquesia, por lo que se realizó una colonoscopia en que se encontró abundante cantidad de líquido hemático de color café oscuro en el lumen, adherido a la mucosa (Figura 1), múltiples pólipos en el colon (Figura 2). Además, se visualizó una formación polipoidea de $18 \mathrm{~mm}$ de diámetro, elevada, pulsátil, con una úlcera en el ciego, cubierta de fibrina en su superficie.

El paciente presentó, además, varios episodios de me-

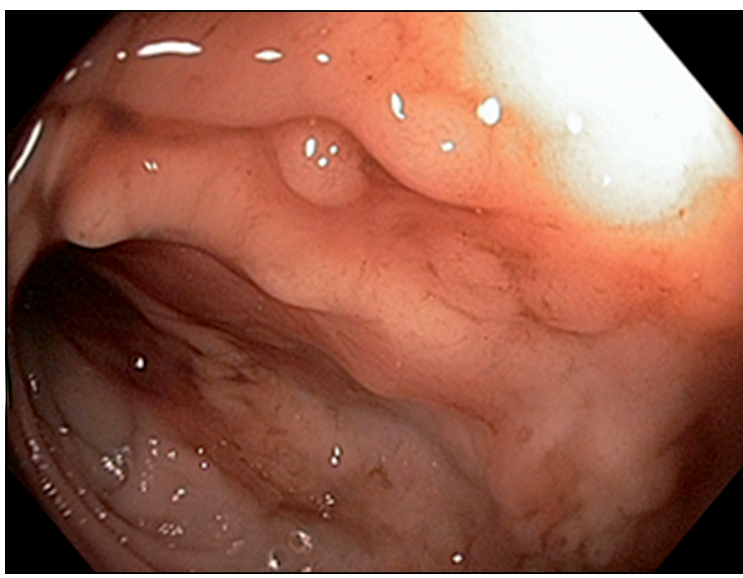
lena posterior a la colonoscopia. Recibió tratamiento con omeprazol en infusión continua y transfusión de glóbulos rojos. Fue sometido a una endoscopia digestiva alta que mostró una gastropatía eritematosa leve sin rastros de sangrado, con múltiples pólipos en el duodeno. Al continuar con melena, el niño se sometió a una segunda laparotomía en donde se encontró líquido libre de aproximadamente $50 \mathrm{cc}$ en cavidad abdominal, además de una masa de $5 \mathrm{~cm}$ de diámetro (Figura 3) parcialmente obstructiva que se extendía hasta la serosa, de consistencia dura e irregular a $90 \mathrm{~cm}$ del ángulo de Treitz. Además, se evidenciaron múltiples ganglios hasta $3 \mathrm{~cm}$ de diámetro en la raíz del mesenterio, mesocolon y colon transverso. Se realizó una resección intestinal con entero-entero anastomosis más hemicolectomía derecha, procedimiento sin complicaciones. El análisis histopatológico de la pieza resecada de la masa de intestino delgado (íleon) y de colon se informó con focos de hiperplasia folicular linfoide, infiltrado inflamatorio polimorfonuclear de la mucosa, submucosa y plano muscular. La pared de íleon presentaba áreas de ulceración de la mucosa, con un importante proceso inflamatorio crónico granulomatoso de todas las capas, con múltiples células gigantes, además de un infiltrado polimorfonuclear eosinófilo moderado, disperso, al igual que en el colon. La tinción de Ziehl Neelsen fue negativa. Con tinción hematoxilina-eosina se identificaron en la mucosa estructuras larvarias, filariformes. Dentro del infiltrado granulomatoso también se identificaron larvas y huevos de un nemátodo estructuralmente compatibles con $S$. stercoralis (imagen no disponible).

El paciente se manejó en un principio en terapia intensiva con terapia antimicrobiana con ceftriaxona y metronidazol, aporte de soluciones intravenosas y nutrición parenteral, evolucionando en forma favorable. Por los hallazgos en el estudio histopatológico recibió tratamiento con ivermectina $200 \mu \mathrm{g} / \mathrm{kg}$ al día por cinco días. La respuesta clínica fue satisfactoria, normalizando las características de las deposiciones. Tras una estancia de 
12 días se dio de alta en buenas condiciones, asintomático y con parámetros de laboratorio normales.

\section{Discusión}

La estrongiloidosis, al igual que otras infecciones tropicales, afecta principalmente a las poblaciones menos privilegiadas y vulnerables del mundo ${ }^{3}$.

La infección intestinal, en muchas ocasiones asintomática, puede adquirir características de gravedad en pacientes con inmunosupresión o con terapia con corticoesteroides, dada su capacidad de alterar la función eosinofílica ${ }^{5}$.

La fisiopatología de la estrongiloidosis explica las distintas formas de presentación de esta enfermedad, es por eso que resulta imprescindible conocer el ciclo biológico del parásito. La larva filariforme de $S$. stercoralis, también denominada L3, es la forma infectante para el ser humano. La larva penetra la piel alcanzando los pulmones a través de la circulación. Al ascender por el árbol respiratorio alcanza la faringe y posteriormente llega al intestino delgado con la deglución. A este nivel madura convirtiéndose en una hembra partenogenética (capaz de reproducirse por sí misma) la que coloca sus huevos en la pared abdominal. Los huevos eclosionan internamente dando lugar a la larva de primer estadio, también llamada larva rabditiforme o L1, que se elimina por las heces. Ya en el suelo se desarrolla directamente a segundo estadio (L2) y tercer estadio (L3) o larva filariforme. Esta última se convierte en ejemplares adultos masculinos y femeninos, quienes a su vez libremente pueden reproducirse produciendo L1 que pasan a L3 completándose el ciclo. Además, en el intestino el estadio L1 puede convertirse en estadio L3, pudiendo la larva penetrar en la mucosa intestinal alcanzando nuevamente los pulmones o el intestino delgado, estableciendo así el ciclo de repetidas infecciones endógenas que conlleva a la infección crónica ${ }^{6}$.

La fuente infectante para el hospedero humano son los suelos húmedos y sombreados contaminados con heces que contienen larvas rabditiformes, las que transformadas en filariformes, por contacto directo con la piel, desencadenan el cuadro clínico.

En la estrongiloidosis, la reacción inmune frente al parásito es poco conocida, existiendo escasa información científica referente al papel de ciertas citoquinas.

La entrada del parásito helminto dirige la activación de las células dendríticas en los tejidos periféricos, de modo que los linfocitos T naive (vírgenes) se diferencian en células T helper 2 (Th2). Los antígenos de reacción cruzada de los helmintos estimulan la participación de las células $\mathrm{T}$ reguladoras naturales $\left(\mathrm{T}_{\mathrm{REG}}\right)$, las mismas que impiden la acción de IL-5, secretada por Th2 y que controlan la respuesta excesiva de éstas células.
En el estado infeccioso crónico los macrófagos adquieren un fenotipo activador alternativo (AAM) que modifica la respuesta de los linfocitos Th2 reduciendo la IL-5 e incrementando la IL-10. Esto hace que los linfocitos B no generen IgE, sino IgG4. Además, transforman el factor de crecimiento $\beta$ (TGF- $\beta$ ), todo lo anterior mediado por una respuesta celular menor ${ }^{12}$.

La estrongiloidosis no complicada debe tratarse en forma oral con ivermectina $200 \mathrm{mcg} / \mathrm{kg}$, una vez al día por uno o dos días, lo cual es, al menos, tan efectivo y mejor tolerado que tiabendazol, $25 \mathrm{mg} / \mathrm{kg}$, dos veces al día por tres días, y más efectivo que albendazol, $400 \mathrm{mg}$, dos veces al día, por tres días ${ }^{8}$.

La visualización endoscópica ha sido reconocida como una importante herramienta diagnóstica en la estrongiloidosis debido a que el parásito invade el duodeno. Sin embargo, se han descrito casos en que los hallazgos endoscópicos e histopatológicos no son métodos confiables para confirmar esta patología ${ }^{9}$. Los exámenes coproparasitológicos tienen poca sensibilidad en el diagnóstico de esta helmintiasis.

El parasitismo es un problema frecuente, pues la morbilidad primaria y secundaria asociada como la anemia crónica y el sangrado digestivo no deben ser desestimados. La pobreza constituye un factor riesgo determinante para todo tipo de parasitosis, es por esto que se encuentra una elevada prevalencia países en vías de desarrollo. La estigmatización, la falta de acceso a los servicios de salud y una escasa tendencia a la búsqueda de atención sanitaria explican que las parasitosis evolucionen con frecuencia en ausencia de tratamiento y que en las poblaciones con recursos escasos los casos de morbilidad grave sean comunes ${ }^{10}$.

\section{Conclusión}

Consideramos que ante un caso como el descrito, debemos aumentar el nivel de sospecha para la estrongiloidosis, teniendo en cuenta que países como el nuestro es endémico en varias parasitosis.

La comunidad de Sarayacu es un territorio de difícil acceso a los servicios de salud. Esto sumado a una escasa tendencia a la búsqueda de atención sanitaria explican que, como lo evidenciado en el caso presentado, una parasitosis de este tipo evolucione tan ampliamente. No existen datos epidemiológicos precisos sobre las enfermedades parasitarias en nuestro país, por lo que es de suma importancia que se investigue este tipo de patologías en especial en poblaciones que viven en una situación de pobreza extrema. Se deben encaminar los esfuerzos para establecer medidas de control de las parasitosis integrando medidas de intervención dirigidas contra las enfermedades desatendidas como la estrongiloidosis. 
Agradecimiento. Al personal de salud implicado en este caso.

\section{Resumen}

Las parasitosis intestinales son una patología frecuente en la consulta pediátrica, constituyendo un problema importante de salud pública. Actualmente, su epidemiología ha variado en países como Ecuador en el que existen poblaciones y comunidades en las que los servicios de salud son escasos o de difícil acceso. Comunicamos el caso de un niño de tres años con desnutrición crónica, proveniente de Sarayacu, provincia de Pastaza, Ecuador, área endémica de parasitosis, que presentó una hemorragia digestiva baja asociado a anemia crónica y eosinofilia. Se realizó una hemicolectomía derecha tras encontrar en la colonoscopia múltiples masas en intestino delgado y de colon. El análisis histopatológico demostró una invasión peritoneal de larvas filariformes de Strongyloides stercoralis.

\section{Referencias bibliográficas}

1.- Hizal G, Ozen H. Gastrointestinal bleeding in children. Journal of Pediatric Sciences. 2011; 3: e100. Disponible en: http://www. pediatricsciences.com/article/view/1050000293/ pdf_122 (Fecha de acceso: 1 de mayo de 2015).

2.- Boyle J T. Gastrointestinal bleeding in infants and children. Pediatr Rev 2008; 2: 39-52.

3.- Swanson S J, Phares C R, Mamo B, Smith K E, Cetron M S, Stauffer W M. Albendazole therapy and enteric parasites in United States-bound refugees. N Engl J Med 2012; 366: 1498-507.

4.- Farthing M, Fedail S. WGO Practice guideline management of strongyloidiasis. WGO 2004; Disponible en: http://www. worldgastroenterology.org/assets/downloads/ en/pdf/guidelines/15_management_ strongyloidiasis_en.pdf (Fecha de acceso: 8 de mayo de 2015).

5.- Llagunes J, Mateo E, Peña J J, Carmona P, de Andrés J. Hiperinfección por Strongyloides stercoralis. Med Intensiva 2010; 34: 353-6.

6.- Martínez-Vázquez C, González-Mediero G, Núñez M, Pérez S, García-Fernández J M, Gimena B. Strongyloides stercoralis en el sur de Galicia. An Med Interna 2003; 20: 477-9.

7.- Carrada-Bravo T. Strongyloides stercoralis: Ciclo vital, cuadros clínicos, epidemiología, patología y terapéutica. Rev Mex Patol Clin 2008; 55: 88-110. Disponible en: http://www. medigraphic.com/pdfs/patol/pt-2008/pt082f.pdf (Fecha de acceso: 3 de junio de 2015).

8.- Vélez J, Giraldo M. Fracaso del tratamiento de estrongiloidiasis con ivermectina. Infect. 2007; 11: 110-20.

9.- Kishimoto K, Hokama A, Hirata T, Ihama Y, Nakamoto M, Kinjo N, et al. Endoscopic and histopathological study on the duodenum of Strongyloides stercoralis hyperinfection. World J Gastroenterol 2008; 14: 1768-73.

10.- Feldmeier H, Heukelbach J. Parasitosis epidérmicas: un problema desatendido asociado a la pobreza. Boletín de la Organización Mundial de la Salud. 2009; 87: 81-160. Disponible en: http://www.who.int/bulletin/ volumes/87/2/07-047308-ab/es/index.html. (Fecha de acceso: 25 de julio de 2015).

11.- Organización Mundial de la Salud. Soiltransmitted helminth infections. Marzo 2016. Disponible en: http://www.who.int/mediacentre/ factsheets/fs366/en/ (Fecha de acceso: 12 de agosto de 2015)

12.- Maizels R M, Yazdanbakhsh M. Inmune regulation by helminth parasites: cellular and molecular mechanisms. Nat Rev Immunol 2003; 3: 733-44. 